\title{
PREVALÊNCIA DE ALZHEIMER EM IDOSOS NAS INSTITUIÇÕES DE LONGA PERMANÊNCIA DA CIDADE DE CAJAZEIRAS-PARAÍBA
}

\author{
ALZHEIMER PREVALENCE IN ELDERLY IN LONG-STAY \\ INSTITUTIONS IN THE CITY OF CAJAZEIRAS-PARAÍBA
}

\author{
Layana Cartaxo Oliveira ${ }^{1}$ \\ Dandara Dias Cavalcante Abreu $^{2}$ \\ Alexsandra Laurindo Leite ${ }^{3}$ \\ Jéssica Alves Moreira ${ }^{4}$
}

RESUMO: Objetivo: Esta pesquisa teve como objetivo investigar a prevalência de Alzheimer em idosos nas Instituições de Longa Permanência da cidade de Cajazeiras - PB. Método: Trata-se de um estudo básico, de campo, com abordagem exploratória, descritiva, de caráter quantitativo. Foi realizada nas instituições do município supracitado, tendo como público-alvo os idosos que as frequentam. A coleta de dados foi realizada através dos prontuários dos idosos fornecidos pelas direções das instituições. Por meio desse instrumento de coleta, foi possível identificar a quantidade de pacientes portadores de Alzheimer, com a finalidade de atender aos objetivos do estudo. O conhecimento dessas informações embasou a análise e interpretação correta dos dados obtidos. Resultados e discussão: Foram estudados 55 idosos dos quais 22\% (12) eram diagnosticados com Alzheimer e 78\% (43) não possuíam a patologia. Com relação aos idosos diagnosticados com Alzheimer, $83 \%(n=10)$ eram mulheres, $58 \%(n=7)$ eram analfabetos. Acerca do tempo que os idosos conviviam nas Instituições de Longa Permanência, $42 \%$ estão morando nas instituições há mais de 5 anos. Idosos com idade superior a 80 anos foi a maior faixa etária encontrada. A maioria dos idosos diagnosticados com a doença de Alzheimer possuía algum tipo de doença crônica, como a Hipertensão. Observou-se que dos 12 idosos diagnosticados com Alzheimer, todos realizavam alguma atividade cognitiva (100\%) e, desses, 8 (66\%) não realizavam atividades físicas. Conclusão: Foi possível concluir, a partir das análises dos resultados obtidos, que existem casos de Alzheimer nas instituições. Como também, que a maioria dos participantes identificados com a patologia não realiza atividades físicas,

\footnotetext{
${ }^{1}$ Biomédica.

2 Biomédica. Docente da Faculdade Santa Maria.

${ }^{3}$ Biomédica. Docente da Faculdade Santa Maria.

${ }^{4}$ Biomédica. Docente da Faculdade Santa Maria.
} 
isso contribui para que a doença aconteça de forma mais rápida. O grau de escolaridade, a faixa etária e as doenças crônicas também estão associadas a incidência dessa doença nos idosos.

Palavras chave: Função cognitiva. Memória. Qualidade de vida.

ABSTRACT: Objective: Objective: This research aimed to investigate the prevalence of Alzheimer's in the elderly in Long Term Care Institutions in the city of Cajazeiras - PB. Method: This is a basic, field study, with an exploratory, descriptive, quantitative approach. It was carried out in the institutions of the aforementioned municipality, with the target audience of the elderly who attend them. Data collection was performed through the medical records of the elderly provided by the institutions' directors. Through this collection instrument, it was possible to identify the number of patients with Alzheimer's, in order to meet the objectives of the study. The knowledge of this information supported the analysis and correct interpretation of the data obtained. Results and discussion: 55 elderly people were studied, of which $22 \%$ (12) were diagnosed with Alzheimer's and 78\% (43) did not have the pathology. The most commonly found level of education was illiteracy, totaling 7 (58\%) patients. Regarding gender, there was a prevalence of women with $83 \%$ of the elderly diagnosed with Alzheimer's (10 occurrences). Regarding the time that the elderly live in Long Term Care Institutions, $42 \%$ have been living in the institutions for more than 5 years. Elderly over 80 years old was the largest age group found. Most elderly people diagnosed with Alzheimer's disease had some type of chronic disease, such as hypertension. It was observed that of the 12 elderly people diagnosed with Alzheimer's, all performed some cognitive activity (100\%) and, of these, 8 (66\%) did not perform physical activities. Conclusion: It was possible to conclude, from the analysis of the results obtained, that there are cases of Alzheimer's in the institutions. As well, that the majority of participants identified with the pathology do not perform physical activities, this contributes to the disease to happen more quickly. Education level, age group and chronic diseases are also associated with the incidence of this disease in the elderly.

Keywords: Cognitive Function. Memory. Quality of life. 


\section{INTRODUÇÃO}

A doença de Alzheimer pode ser definida como uma patologia evolutiva que possui a capacidade de atingir várias funções mentais, como a perda da memória, expressão, raciocínio e entendimento. Além disso, progride com o passar do tempo, gerando dependência, principalmente na fase final da doença, uma vez que o paciente já não consegue realizar as atividades do cotidiano sem a ajuda de um terceiro. Essa situação causa uma intensa mudança no dia a dia da pessoa com a doença, bem como na dos cuidadores e familiares, no que concerne à qualidade de vida e, principalmente, aos desgastes físicos e emocionais envolvidos (SANTOS; BORGES, 2015).

No cuidado com pessoas idosas portadoras de doenças neurodegenerativas, os familiares e cuidadores precisam ter disciplina, uma vez que o diagnóstico traz consigo mudanças significativas em vários parâmetros (sociais, financeiros e emocionais). Para tanto, faz-se necessário a prática de diversas atitudes para cuidar melhor do paciente, tais como: dedicar a maior parte do tempo a eles, ajudando principalmente na alimentação e na higiene, pois, na maioria das vezes, isso constitui sentimentos de temor, riscos e perdas para a pessoa acometida com a doença. Através da disciplina, o cuidador consegue sabedoria para conviver e ajudar nas dificuldades que a doença proporciona, como a mudança de comportamento e dependência, bem como adaptação a nova rotina (ILHA, et al., 2015).

A doença pode se manifestar de dois modos: a primeira, início tardio, ou seja, a forma mais popular, que é adquirida após os 60 anos de idade; e a segunda, familiar, que ocorre antes dos 60 anos, pois possui um vigoroso poder hereditário. Contudo, as duas formas são caracterizadas pelos mesmos sintomas (FALCO; et al., 2015).

De acordo com os processos evolutivos da doença, podemos dividi-los em três categorias: inicial, moderado e severo. É válido ressaltar que as mudanças auditivas estão aliadas ao obstáculo da comunicação, que vão progredindo para 
fases mais elevadas, como a dificuldade na alimentação (no ato de mastigar e deglutir os alimentos), tornando o paciente dependente de outras pessoas para se alimentar e realizar atividades básicas diárias (TAVARES; CARVALHO, 2012). Para esta patologia, o diagnóstico é de pretensão clínica. A família e a equipe multidisciplinar são relevantes para auxiliar o médico no diagnóstico da patologia. Dentre os inúmeros exames que devem ser realizados, os principais são: os laboratoriais, os de neuroimagens e os neuropsicológicos (Teste do Desenho do Relógio e Teste de Fluência Verbal) (POLTRONIERE; et al., 2011).

Além de ser uma doença de relevante magnitude e, ainda incurável, é caracterizada pelo alto custo financeiro e demandas exaustivas de cuidados com os pacientes, sendo, portanto, um grande desafio para todos os envolvidos. Desse modo, o presente estudo tem o objetivo de investigar a prevalência de Alzheimer em idosos nas Instituições de Longa Permanência da cidade de Cajazeiras - PB.

A pesquisa justifica-se pela ausência de informações sobre a temática abordada. Busca, ainda, obter respostas sobre quais os principais impactos na qualidade de vida dos pacientes diagnosticados com Alzheimer e de seus familiares, além de contribuir para melhoria na saúde e na qualidade de vida desses idosos. Assim sendo, ela contribuirá com futuros estudos bibliográficos na comunidade acadêmica e científica que também envolvam a temática em questão.

\section{MATERIAIS E MÉTODOS}

Esta pesquisa consiste em um estudo exploratório, de aspectos quantitativos que possibilitou desenvolver o tema partindo do intento de investigar a prevalência de Alzheimer em idosos.

O estudo foi realizado em três instituições de longa permanência, instituições de caráter residencial criadas com a finalidade de servir de domicílio coletivo para pessoas com idade igual ou superior a 60 anos, com ou sem suporte familiar, oferecendo-os condições de liberdade, dignidade e cidadania, todas localizadas no município de Cajazeiras, no estado da Paraíba. A amostra foi constituída por 55 
(cinquenta e cinco) idosos, de ambos os sexos, vinculados às instituições pesquisadas, que foram diagnosticados com ou não com a doença de Alzheimer no período compreendido desde a data de ingresso dos idosos nas instituições até o mês de abril de 2018.

A coleta de dados foi realizada através dos prontuários dos idosos fornecidos pela direção da instituição, a fim de avaliar a quantidade de pacientes portadores de Alzheimer. Foram avaliados todos os idosos que convivem nas instituições de longa permanência, destacando-se aqueles que possuem a patologia. Foram coletados dados através de formulários com questões como sexo, faixa etária, prevalência de doenças crônicas, escolaridade, tempo de instituição, prática de atividades físicas e cognitivas.

Os dados coletados também foram relacionados ao tempo em que os idosos estão vinculados à instituição, bem como às práticas desenvolvidas (atividades lúdicas para a estimulação cognitiva e atividades físicas regulares, por exemplo), correlacionado se colaboram ou não para o aparecimento e desenvolvimento da patologia em estudo. Na pesquisa não foram indicados dados pessoais como nome e endereço dos estudados, segundo o que preconiza a Resolução $n^{\circ} 466 / 12$, de 12 de dezembro de 2012, do Conselho Nacional de Saúde, que visa assegurar os direitos e os deveres dos participantes da pesquisa, da comunidade científica e do Estado.

Os dados foram coletados por meio dos formulários e organizados no próprio instrumento de coleta de dados. Após serem coletados, foram explorados de forma estatística. Eles foram estruturados em um banco de dados eletrônico do aplicativo Microsoft Excel, sendo codificados, tabulados e apresentados em forma de tabelas, quadros e gráficos com suas respectivas distribuições percentuais. A discussão foi realizada com base na revisão bibliográfica para subsidiar e fundamentar a pesquisa.

A coleta ocorreu nos meses de março e abril de 2018, imediatamente após a aprovação do Comitê de Ética em Pesquisa (CEP) da Faculdade Santa Maria (FSM), cujo parecer de aprovação é de número 2.615.812. 


\section{RESULTADOS E DISCUSSÃO}

A pesquisa analisou a prevalência de Alzheimer em idosos nas Instituições de Longa Permanência da cidade de Cajazeiras-PB, cuja a amostra foi constituída por 55 idosos. De todos os idosos pesquisados, observou-se que $22 \%$ (12) foram diagnosticados com a doença de Alzheimer e $78 \%$ (43) não apresentaram diagnóstico para a mesma.

Lini et al. (2014) realizaram uma investigação descritiva com 250 idosos institucionalizados no município de Passo Fundo, Rio Grande do Sul. Nesse estudo, evidenciou-se que, dos 250 idosos institucionalizados, 27,5\%, a minoria, tinham a doença de Alzheimer. Esse resultado vai ao encontro da presente pesquisa, pois também a minoria 22\% (12) foram diagnosticados com a patologia em questão. Contrapondo-se a estas, Mello et al. (2012), apresentaram que dos seus 44 idosos avaliados, 38,6\% (17) apresentavam demências, sendo o Alzheimer a mais prevalente, pois revelou que $59 \%$ (26) dos idosos participantes possuíam, representando a maioria.

Com relação ao sexo, $17 \%$ (2) dos idosos que foram diagnosticados com a doença de Alzheimer pertencem ao sexo masculino e $83 \%$ (10) são do sexo feminino. Daltin (2016), que obteve seu estudo a partir do Programa Municipal de Atenção ao Idoso (PROMAI), observou que a prevalência da população observada era do sexo feminino, $66(81,5 \%)$, e que $15(18,5 \%)$ eram do sexo masculino. A mesma predominância feminina também aconteceu no presente estudo.

Geralmente as mulheres idosas lidam mais com as doenças, diferentemente dos homens que, muitas vezes, têm óbitos mais precoce, não chegando a conviver tanto com as doenças crônicas como as mulheres. Isso pode estar relacionado ao fato que as mulheres sempre estão à procura de serviços da saúde, favorecendo um diagnóstico prévio, aumentando assim, a sobrevida delas. Essa relação aponta que o sexo feminino, em conjunto, possui um tempo de sobrevivência maior que o sexo masculino. Contudo, se os homens não tivessem óbitos por causa de outras 
patologias, o número de Alzheimer seria praticamente igual em ambos os sexos (OLIVEIRA; et al., 2005).

Em relação à escolaridade, 58\% (7) são analfabetos, 34\% (4) possuem apenas o ensino fundamental I ( $1^{\circ}$ ao $4^{\circ}$ ano); e $8 \%$ (1) possui ensino superior, conforme a Tabela 1. Não foi detectado nenhum idoso que apresentasse ensino fundamental II $\left(5^{\circ}\right.$ ao $\left.9^{\circ}\right)$, nem ensino médio completo.

\section{Tabela 1 - Grau de escolaridade dos idosos participantes da pesquisa.}

Analfabeto

\begin{tabular}{c|c}
\hline 7 & $58 \%$ \\
\hline 4 & $34 \%$ \\
\hline 1 & $8 \%$ \\
\hline 12 & $100 \%$ \\
\hline
\end{tabular}

Fundamental I $\left(1^{\circ}\right.$ ao $4^{\circ}$ ano $)$

Ensino Superior

TOTAL 12 $100 \%$

Fonte: Primária (2018).

Nos estudos realizados por Lira e Santos (2012), feito no ambulatório do Centro Geriátrico do Hospital Santo Antônio da cidade de Salvador, Bahia, verificouse que $3(10 \%)$ dos idosos acometidos com a doença de Alzheimer eram analfabetos, 15 (50\%) tinham o $1^{\circ}$ grau incompleto, 6 (20\%) tinham o $1^{\circ}$ grau completo, $4(13,3 \%)$ tinham o $2^{\circ}$ grau completo e $2(6,7 \%)$ tinham o ensino superior, ou seja, a maior parte dos indivíduos estudados frequentou a escola por até oito anos. O que vai de encontro aos estudos de Azevedo et al. (2010), no Centro de Reabilitação Regional de Araraquara (CRRA), uma vez que ele mostra que, dos 27 sujeitos analisados, 12 (44,4\%) eram analfabetos, concordando, pois, com o atual estudo. Rocha, Klei e Paschoalotti (2014) corroboram que o baixo grau de escolaridade possui influência com as funções cognitivas que se apressam durante a velhice.

A escolaridade é de suma importância, pois o baixo grau de instrução aumenta as chances de adquirir qualquer tipo de demência. Isso pode dificultar a compreensão da patologia, o entendimento e a coleta das informações pelos profissionais da saúde. Além disso, é um fator que prejudica os testes de rastreio como, por exemplo, o Mini-mental e o Teste do relógio (GRATÃO; et al., 2015).

De acordo com as informações reveladas pela tabela 2 , com relação ao tempo que os idosos convivem nas instituições de longa permanência, $17 \%$ (2) frequentam de 6 meses a 1 ano; 33\% (4) frequentam de 1 a 3 anos; 8\% (1) 
frequentam de 3 a 5 anos e 42\% (5) convivem a mais de 5 anos na instituição. Nenhum deles frequenta a instituição a um tempo inferior a 6 meses.

Tabela 2 - Tempo que os idosos frequentam a instituição de longa permanência.

\begin{tabular}{l|cc} 
De 6 meses a 1 ano & 2 & $17 \%$ \\
\hline De 1 a 3 anos & 4 & $33 \%$ \\
\hline De 3 a 5 anos & 1 & $8 \%$ \\
\hline A mais de 5 anos & 5 & $42 \%$ \\
\hline TOTAL & $\mathbf{1 2}$ & $\mathbf{1 0 0 \%}$ \\
\hline
\end{tabular}

Fonte: Primária (2018)

Nos estudos de Oliveira e Rozendo (2014), em Instituições de Longa Permanência para Idosos de caráter filantrópico de uma capital da região Nordeste, constatou-se que o tempo de admissão em instituições dessa natureza varia entre seis meses e 12 anos. Para ele, o alto número de idosos e a longa duração de vida da população acrescenta uma maior dificuldade na saúde dos idosos. Sendo assim, existe uma falta de familiares/cuidadores para eles, crescendo mais ainda a demanda das instituições.

Silva et al. (2017), em um trabalho realizado na cidade de Itambé-BA, retratou que, atualmente, um problema crescente no Brasil é o da institucionalização, devido as famílias não terem tempo para cuidar dos idosos. A convivência nessas instituições pode apresentar pontos negativos no que se diz respeito à saúde, qualidade de vida e na utilidade do paciente, o que justifica os dados encontrados na pesquisa. Segundo Borges e Mendes (2015), o tempo de vivência dos idosos nas instituições está diretamente relacionado aos problemas na saúde mental, como a depressão e deficiência nas funções cognitivas, ou seja, condições que deixam o idoso fragilizado.

De acordo com o gráfico 1, observou-se que dos idosos que foram acometidos com Alzheimer e que convivem nas instituições de longa permanência, 25\% (3) estavam entre a faixa etária de 60 a 70 anos; 34\% (4) entre 70 a 80 anos; $25 \%$ (3) se encontravam na faixa etária de 80 à 90 anos; 8\% (1) deles exibia faixa etária de 90 à 100 anos e 8\% (1) possuía idade acima de 100 anos. 
Gráfico 1: Faixa etária dos idosos que frequentam a instituição de longa permanência.

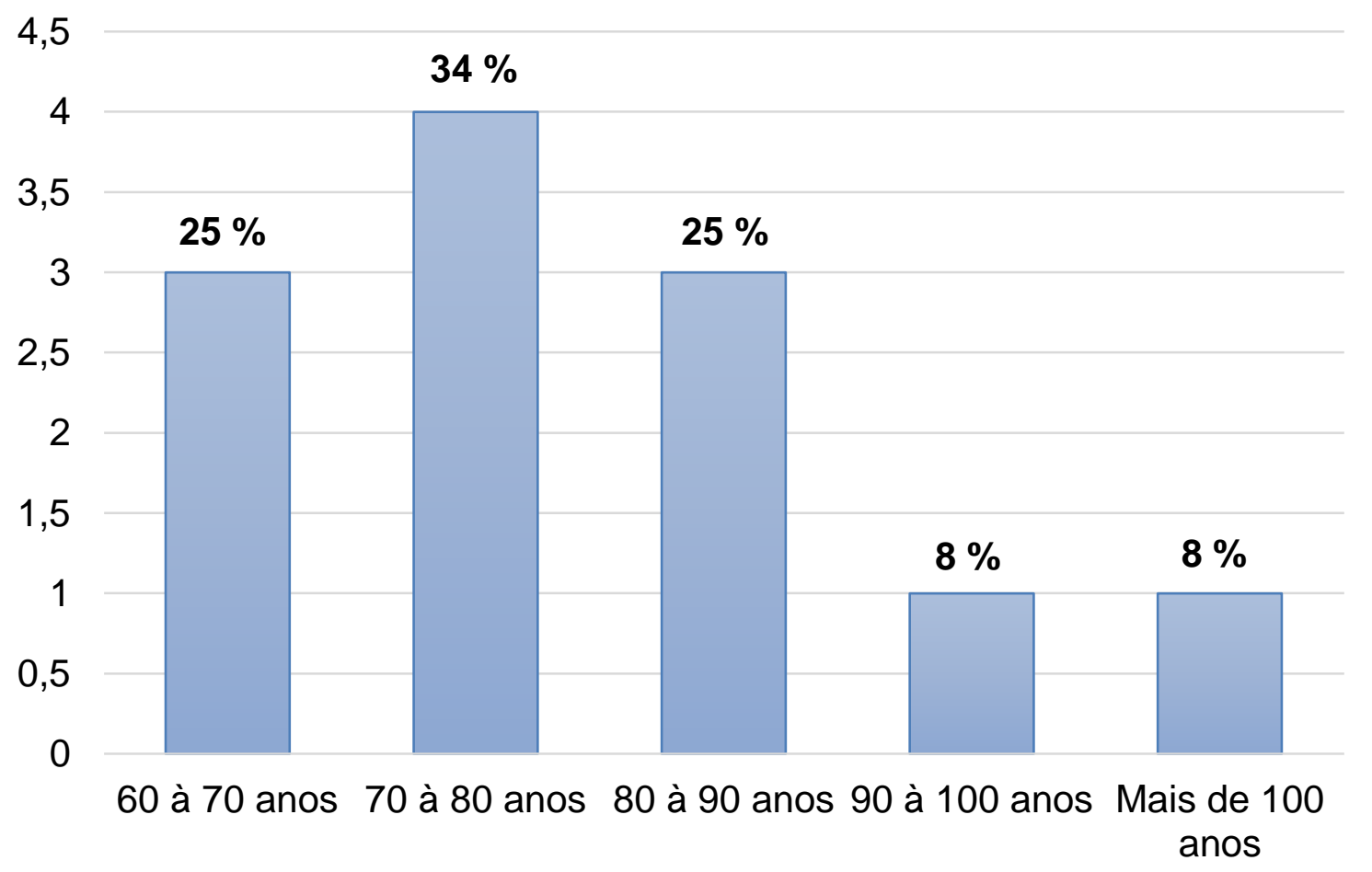

Fonte: Primária.

Oliveira e Mattos (2012) através de seus estudos observou que houve a predominância da faixa etária entre 70 a 79 anos com 37,7\% da sua amostra (57), logo em seguida prevaleceu os indivíduos com mais de 80 anos com 35,1\% (53), demonstrando uma contraposição com nossos presentes dados, onde os indivíduos com mais de 80 anos prevaleceram com $41 \%$ (5).

Concordando também com a pesquisa de Oliveira e Tavares (2014), onde sua análise foi realizada em instituições de longa permanência no interior de São Paulo, e foi observado que $47,4 \%$ (41) eram de idosos com mais de 80 anos, podendo ser justificado por causa do aumento dos nascimentos ocorridos em uma população anteriormente, diminuição da mortalidade e a melhoria dos avanços tecnológicos na saúde. Já para Tonholi e Oltramari (2017) descreveu que a faixa etária não obteve relevância para o seu estudo, pelas diferentes idades. 
Segundo o gráfico 2, dos 12 diagnosticados com Alzheimer, 9 possuíam algum tipo de doença crônica, predominando a hipertensão com $45 \%$ (4) dos casos; logo em seguida Diabetes Mellitus com 11\% (1); e aqueles que têm mais de uma patologia, como hipertensão e Diabetes Mellitus com 22\% (2); hipertensão e Epilepsia com 11\% (1); e por fim, hipertensão e problemas cardíacos com 11\% (1) dos casos.

Gráfico 2 - Relação de Alzheimer com doenças crônicas.

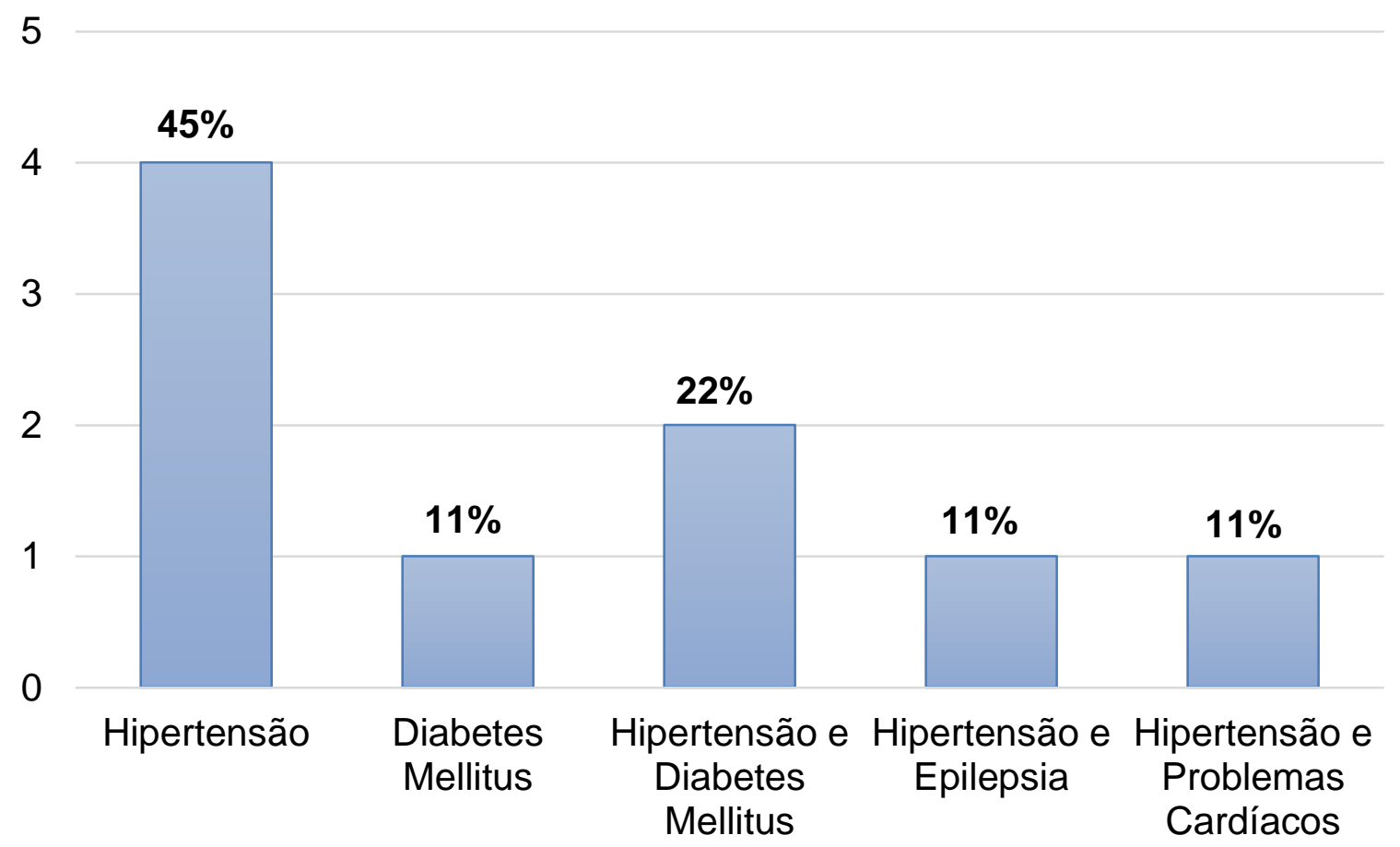

Fonte: Primária (2018).

Conforme os estudos de Pereira (2012), que foi realizado com idosos institucionalizados na cidade de João Pessoa-PB, houve a prevalência da hipertensão com $20(71,4 \%)$ da sua população, estando de acordo com nossos resultados no gráfico 2. Para ele, a hipertensão é um grande problema de saúde pública, dados os riscos e a dificuldade para um bom controle.

Segundo Santos et al. (2017), o Diabetes Mellitus possui um alto fator de risco para o desenvolvimento da doença de Alzheimer, podendo ser até chamada de Diabetes Mellitus Tipo 3, por causa de sua semelhança com as anomalias 
histopatológicas, moleculares e bioquímicas. O Diabetes Mellitus Tipo 2 produz estresse oxidativo, podendo-se disseminar por todo o cérebro que possui uma alta sensibilidade ao mesmo, visto que consome uma boa quantidade de oxigênio e possui ácidos graxos, sendo motivo dos ataques dos radicais livres, atingindo as células, principalmente os neurônios, causando a morte programada da célula (apoptose).

Verificou-se que todos os idosos que possuem Alzheimer nas instituições de longa permanência realizam alguma atividade para estimulação cognitiva, porém apenas $4(34 \%)$ idosos realizam atividade física diariamente e 8 (66\%) não realizam nenhum tipo de atividade física. Nossos dados contrapõem com os achados das análises realizadas por Queiroz (2010), ao analisar 12 idosos, dos quais $8(80 \%)$ da sua população realizavam exercícios físicos diariamente através de ginásticas, caminhadas e fisioterapias.

Carvalho et al. (2016), relatam que quando o idoso é diagnosticado com a doença de Alzheimer, o tratamento começa através de medicamentos para melhorar os sinais e sintomas. Entretanto, existe o tratamento não farmacológico com a estimulação cognitiva através de atividades mentais, como os exercícios de memória, a realização de palavras cruzadas, leitura, entre outros. Com isso, a qualidade de vida do indivíduo irá melhorar, como também retardará o processo patológico.

Diversas atividades diárias como realizar cálculos matemáticos, ler jornais e revistas, tocar algum instrumento musical e assistir televisão foram relacionados a estímulo da cognição, diminuindo assim os fatores de risco para o Alzheimer (LOPES; et al., 2011).

Quanto mais atividade física, menor o risco para adquirir a patologia, pois é produzida uma substância chamada de Fator Neutrófico Derivado do Cérebro (BDNF), que estimula as sinapses, a neurogênese, melhora o desempenho cerebral, reduzindo o acúmulo da proteína Beta-amilóide no cérebro, preservando, assim, o desenvolvimento da doença de Alzheimer (NASCIMENTO, 2009). 


\section{CONSIDERAÇÕES FINAIS}

A partir das análises dos resultados obtidos, foi possível concluir que existem casos de Alzheimer nas instituições observadas. Constatou-se, por sua vez, que a maioria desses participantes não realizam atividades físicas, fazendo com que a doença ocorra de forma mais rápida. Também se assinalou uma tênue relação entre a faixa etária e as doenças crônicas à ocorrência de Alzheimer.

São vários os impactos causados na vida do paciente acometido com a doença, porém o principal é a dependência, pois eles não conseguem exercer seu dia a dia com qualidade, seja para se alimentar, andar ou para realizar as necessidades higiênicas sozinhos, necessitando da ajuda de familiares e/ou cuidadores para auxiliá-los.

Portanto, faz-se necessário ocorrências de campanhas para explicarem a gravidade dessa patologia e a importância dos exercícios cognitivos e físicos para a prevenção da doença. Além disso, é imensa a necessidade da realização de exames para um diagnóstico precoce, como também, é essencial a universalização desses serviços para o público de baixa renda.

\section{REFERÊNCIAS BIBLIOGRÁFICAS}

AZEVEDO, P. G.; LANDIM, M. E.; FÁVERO, G. P.; CHIAPPETTA, A. L. M. L. Linguagem e memória na doença de Alzheimer em fase moderada. Revista CEFAC, São Paulo, v. 12, n. 3, p. 393-399, jun. 2010.

BORGES, A. P. M.; MENDES, G. C. Avaliação cognitiva e de equilíbrio em idosos institucionalizados após intervenção de xbox terapia. Cad. da Esc. de Saúde, Curitiba, v. 1, n. 13, p. 93-104, mar. 2015.

CARVALHO, P. D. P.; MAGALHÃES, C. M. C.; PEDROSO, J. S. Tratamentos não farmacológicos que melhoram a qualidade de vida de idosos com doença de Alzheimer: uma revisão sistemática. Jornal brasileiro de psiquiatria, Rio de Janeiro, v. 65 , n. 4, p. 334-339, dez. 2016.

DALTIN, J. B. Uso de medicamentos em pacientes idosos portadores de doença de Alzheimer. 2016. 111f. Dissertação (Mestrado em Pós-graduação em Saúde Coletiva) Faculdade de Medicina de Botucatu - UNESP, São Paulo, 2016. 
FALCO, A.; CUKIERMAN, D.S.; DAVIS, R.A.H.; REY, N.A. Doença de Alzheimer: hipóteses etiológicas e perspectivas de tratamento. Quím. Nova, São Paulo, v. 39, n. 1, p. 63-80, jan. 2016.

FUZARO JUNIOR, Gilson. Nível de atividade física, estado nutricional e níveis de BACE 1 e BACE 2 em idosos neurologicamente saudáveis e com doença de Alzheimer. 2015. 89 f. Dissertação - (mestrado) - Universidade Estadual Paulista, Instituto de Biociências de Rio Claro, 2015.

GRATÃO, A. C. M; COSTA, A. C; DINIZ, M. A. A. Condições de saúde de idosos e cuidadores em uma instituição de longa permanência para idosos. Rev Enferm UFPE, v. 9, p. 7562-71, 2015.

ILHA, S.; BACKES, D. S.; SANTOS, S. S. C.; GAUTÉRIO-ABREU, D. P.; ZAMBERLAN, C.; BLASI, T. C. Group for family/caregivers of elderly with Alzheimer's: experience of teachers based on the complexity. : experience of teachers based on the complexity. Revista da Rede de Enfermagem do Nordeste, [s.I.], v. 16, n. 4, p. 603, 4 ago. 2015.

KUGA, G. K.; BOTEZELLI, J. D.; GOMES, R. J.; PAULI, J. R.; LEME, J. A. C. A. Hippocampal insulin signaling and neuroprotection mediated by physical exercise in Alzheimer's Disease. Motriz: rev. educ. fis., Rio Claro, v. 23, n. spe, e101608, 2017.

LINI, E.; DORING, M.; MACHADO, V.; PORTELLA, M. Idosos institucionalizados: prevalência de demências, características demográficas, clínicas e motivos da institucionalização. Revista Brasileira de Ciências do Envelhecimento Humano, v. 11, n. 3, 27 dez. 2014.

LIRA, M.; SANTOS, L.C. C. S. Correlação entre função cognitiva e capacidade funcional nos indivíduos com doença de Alzheimer. Cadernos de Pós-Graduação em Distúrbios do Desenvolvimento, São Paulo, v.12, n.2, p. 36-45, 2012.

LOPES, L. C.; ARAÚJO, L. M. Q.; CHAVES, M. L. F.; IMAMURA, M.; OKAMOTO, I. H.; RAMOS, A. M.; SATOMI, E.; STEIN, A. T.; CENDOROGLO, M. S.; SOUZA, A. M. C.; APOLINÁRIO, D.; ANDRADA, N. C. Doença de Alzheimer: Prevenção e Tratamento. Diretrizes Clínicas na Saúde Suplementar - Associação Médica Brasileira e Agência Nacional de Saúde Suplementar, $2011 . \quad$ Disponível em: https://diretrizes.amb.org.br/ans/doenca_de_alzheimer-diagnostico.pdf. Acesso em: 12 abr. 2018.

MELLO, B. L. D. DE; HADDAD, M. DO C. L.; DELLAROZA, M. S. G. Avaliação cognitiva de idosos institucionalizados. Acta Scientiarum. Health Sciences, Maringá, v. 34, n. 1, p. 95-102, jan-jun. 2012.

MENDES, C. F. M.; SANTOS, A. L. S. O cuidado na doença de Alzheimer: as representações sociais dos cuidadores familiares. Saúde soc., São Paulo, v. 25, n. 1, p. 121-132, mar. 2016.

NASCIMENTO, G. L. O exercício físico na prevenção da doença de Alzheimer. Revista saúde integrada, v. 2, n. 3-4, p. 81-106, 2009.

OLIVEIRA, J. M.; ROZENDO, C. A. Instituição de longa permanência para idosos: um lugar de cuidado para quem não tem opção?. Rev. bras. enferm. Brasília, v. 67, n. 5, p. 773-779, out. 2014.

OLIVEIRA, M. F.; RIBEIRO, M.; BORGES, R.; LUGINGER, S. Doença de Alzheimer: perfil neuropsicológico e tratamento. [Trabalho de Licenciatura]. Portugal: Universidade Lusíada do Porto, 2005.

OLIVEIRA, P. B.; TAVARES, D. M. S. Condições de saúde de idosos residentes em Instituição de Longa Permanência segundo necessidades humanas básicas. Rev. bras. enferm. Brasília, 
v. 67, n. 2, p. 241-246, abr. 2014.

POLTRONIERE, S.; CECCHETTO, F. H.; SOUZA, E. N. Doença de Alzheimer e demandas de cuidados: o que os enfermeiros sabem?. Rev. Gaúcha Enferm. Porto Alegre, v. 32, n. 2, p.270278, jun. 2011.

QUEIROZ, G. A. Qualidade de vida em instituições de longa permanência para idosos: considerações a partir de um modelo alternativo de assistência. 2010. 164f. Dissertação de Mestrado em Psicologia, Universidade Federal de São João del-Rei, São João del-Rei, 2010.

ROCHA, J. P.; KLEIN, O. J.; PASQUALOTTI, A. Qualidade de vida, depressão e cognição a partir da educação gerontológica mediada por uma rádio-poste em instituições de longa permanência para idosos. Revista Brasileira de Geriatria e Gerontologia, Rio de Janeiro, v.17. n. 1, p. 115-128, 2014.

SANTOS, A. L. M.; FRAGA, V. G.; MAGALHÃES, C. A.; SOUZA, L. C.; GOMES, K. B. Doença de Alzheimer e diabetes mellitus tipo 2: qual a relação?. Revista Brasileira de Neurologia, v. 53, p. 17-26, 2017.

SANTOS, M. D.; BORGES, S. M. Percepção da funcionalidade nas fases leve e moderada da doença de Alzheimer: visão do paciente e seu cuidador. Rev. bras. geriatr. gerontol., Rio de Janeiro, v. 18, n. 2, p. 339-349, jun. 2015.

SILVA, C. S.; SAMPAIO, L. S.; SAMPAIO, T. S. O. Capacidade funcional de idosos em instituição de longa permanência. Rev. Mult. Psic, v. 11, n. 38, 2017.

TAVARES, T. E.; CARVALHO, C. M. R. G. Características de mastigação e deglutição na doença de Alzheimer. Rev. CEFAC, São Paulo, v. 14, n. 1, p. 122-137, fev. 2012.

TONHOLI, D. F.; OLTRAMARI, G. Prevalência, desempenho cognitivo e funcionalidade de idosos com doença de Alzheimer em instituições de longa permanência de Bento Gonçalves. Pajar, v. 5, n. 1, p. 23-29, 2017. 